\title{
Microscopic investigation of the structure characteristics and wave functions of the five-body hypernucleus $H e_{\lambda}^{5}$
}

\author{
Lia Leon Margolin ${ }^{1}$ \\ ${ }^{1}$ Marymount Manhattan College, Mathematics Department, 221 E $71^{\text {st }}$ St. New York, NY, USA
}

\begin{abstract}
The Parentage Scheme of Summarization to the N-body symmetrized basis construction [1], necessary for the description of the structural characteristics and decay reactions of the hypernuclear and nuclear systems with arbitrary amount of particles, is applied to the solution of five-body problem in hypernuclear physics. Hypernucleus $H e_{\lambda}^{5}$ as a system of four nucleons and one hyperon is investigated by the use of the Hyperspherical Function Method in momentum space. The dependence of the structure characteristics and wave functions on the types of nucleon-nucleon and hyperon-nucleon interaction potentials is studied. Mean square $\lambda-H^{3}$ and $\alpha-\lambda$ distances and the binding energies for the $\mathrm{He}^{4}$ and $H e_{\lambda}^{5}$ are obtained.
\end{abstract}

\section{INTRODUCTION}

The principal information about nucleon-nucleon and hyperon-nucleon interactions may be obtained by the microscopic investigation of the structure and decays of light hypernuclei within the framework of the Hyperspherical Function Method (HFM). Generalized mathematical formalism to the construction of N-particle fully symmetrized hyperspherical functions on the basis of the N-particle hyperspherical functions symmetrized with respect to $\mathrm{N}-1$-particles was introduced in [1]. The microscopic approach to the study of the structure and mesonic decays of four-body hypernucleus $H_{\lambda}^{4}$ by the use of the HFM in momentum space was developed and structure characteristics and decay rates were obtained [2-5]. The binding energy of $H e_{\lambda}^{5}$ was evaluated by the use of the microscopic $\alpha-$ $\lambda$ cluster model [6].

This article, for the first time, investigates Hypernucleus $H e_{\lambda}^{5}$ as a system of four nucleons and one hyperon. The microscopic approach to the solution of five- body problem in hypernuclear physics within the framework of the HFM in momentum representation is developed in section 2 . The binding energies of $H e^{4}$ and $H e_{\lambda}^{5}$ and the mean square $\lambda-H^{3}$ and $\lambda-\alpha$ distances for $H e^{4}$ and $H e_{\lambda}^{5}$ respectively by the use of the different nucleon-nucleon and hyperon-nucleon potentials are obtained and compared with experimental results in section 3 .

\section{Microscopic investigation of $H e_{\lambda}^{5}$}

Hypernucleon $H e_{\lambda}^{5}$ as a system consisting of four identical particles (nucleons) and one particle of a different mass ( $\lambda$-hyperon) can be described by the following equation in momentum space: 
$\left(\mathcal{\varkappa}^{2}+\overline{\mathcal{H}}_{0}^{2}\right) \Psi(\boldsymbol{p}, \boldsymbol{q}, \boldsymbol{r}, \boldsymbol{s})=-2 \mathrm{M} / \hbar^{2} \times$

$\int \Psi(\boldsymbol{p}, \boldsymbol{q}, \boldsymbol{r}, \boldsymbol{s})\left\langle\boldsymbol{p}, \boldsymbol{q}, \boldsymbol{r}, \boldsymbol{s}\left|U_{12345}(\boldsymbol{x}, \boldsymbol{y}, \mathbf{z}, \boldsymbol{w})\right| \boldsymbol{p}^{\prime}, \boldsymbol{q}^{\prime}, \boldsymbol{r}^{\prime}, \boldsymbol{s}^{\prime}\right\rangle$ dpdqdrds

where $\overline{\mathcal{X}}_{0}^{2}=\frac{2 M \varepsilon}{\hbar^{2}}, \varepsilon$ is the binding energy of the system, $\mathcal{X}^{2}=p^{2}+q^{2}+r^{2}+s^{2}$ is the twelve

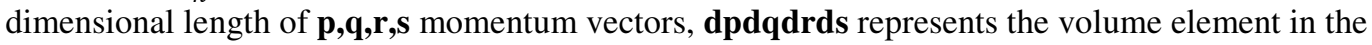
twelve dimensional space, and $\Psi(\boldsymbol{p}, \boldsymbol{q}, \boldsymbol{r}, \boldsymbol{s})$ is a five-body wave function in momentum space which can be expanded in the hyperspherical basis [1]:

$$
\Psi(\boldsymbol{p}, \boldsymbol{q}, \boldsymbol{r}, \boldsymbol{s})=\sum_{\alpha} \varphi_{K_{5} L}^{\alpha}(\varkappa) \Psi_{K_{5} L}^{\alpha}\left(\omega_{\varkappa}\right)
$$

where $\alpha \equiv\left(l_{1}, l_{2}, l_{12}, l_{3}, l_{123}, l_{4}, K_{3}, K_{4}\right), \omega_{\mathcal{\varkappa}}$ is the twelve dimensional solid angle in $\mathbf{p}, \mathbf{q}, \mathbf{r}, \mathbf{s}$ vectorial space with eight angles determining the direction of vectors $\mathbf{p}, \mathbf{q}, \mathbf{r}, \mathbf{s}$ and three angles $\alpha_{\varkappa}, \beta_{\varkappa}, \gamma_{\varkappa}$ related to the lengths of these vectors with the following expressions:

$p=\varkappa \cos \alpha_{\varkappa} \sin \beta_{\varkappa} \sin \gamma_{\varkappa} ; q=\varkappa \sin \alpha_{\varkappa} \sin \beta_{\varkappa} \sin \gamma_{\varkappa} ; r=\varkappa \cos \beta_{\varkappa} \sin \gamma_{\varkappa} ; w=\cos \gamma_{\varkappa}$

Expanding the twelve dimensional plane wave $|\boldsymbol{p}, \boldsymbol{q}, \boldsymbol{r}, \boldsymbol{s}\rangle$ in hypersperical basis and implementing formula (2) in (1) produces the following system of the coupled one- dimensional integral equations:

$$
\begin{aligned}
& \mathcal{\varkappa}^{5}\left(\mathcal{\varkappa}^{2}+\bar{\varkappa}_{0}^{2}\right) \varphi_{K_{5} L}^{\alpha}(\mathcal{\varkappa})=-\frac{2 \mathrm{M}}{\hbar^{2}} \times \\
& \times \sum_{K_{5}^{\prime}} i^{K_{5}^{\prime}-K_{5}} \int d \mathcal{\varkappa}^{\prime} \mathcal{H}^{\prime 6} \varphi_{K_{5}^{\prime} L^{\prime}}^{\alpha^{\prime}}\left(\mathcal{H}^{\prime}\right) \int \rho d \rho J_{K_{5}+5}\left(\varkappa_{\rho}\right) J_{K_{5}^{\prime}+5}\left(\mathcal{H}^{\prime} \rho\right)\left\langle\Psi_{K_{5} L}^{\alpha}\left(\omega_{\rho}\right)\left|U_{1234}\right| \Psi_{\mathrm{K}_{5}^{\prime} \mathrm{L}^{\prime}}^{\alpha^{\prime}}\left(\omega_{\rho}^{\prime}\right)\right\rangle
\end{aligned}
$$

Full wave functions of the $H e_{\lambda}^{5}$ are constructed by combining hyperspherical, radial, and spin-isospin parts:

$$
\Psi(\mathbf{p}, \mathbf{q}, \mathbf{r}, \mathbf{s}\{\boldsymbol{\sigma}, \boldsymbol{\tau}\})=\sum_{K_{5}[\bar{f}] \bar{v}} \varphi_{K_{5} L}^{[\bar{f}] \bar{v}}(\varkappa) \Gamma_{\mathrm{K}_{5} \mathrm{LST}}\left(\omega_{\varkappa}, \boldsymbol{\sigma}, \boldsymbol{\tau}\right)
$$

where

$$
\begin{array}{r}
\Gamma_{\mathrm{K}_{5} \mathrm{LST}}\left(\omega_{\mathcal{\varkappa}}, \boldsymbol{\sigma}, \boldsymbol{\tau}\right)=\frac{1}{\sqrt{h_{\overline{[f]}}}} \sum_{\bar{m}} \Psi_{K_{4} K_{4}}^{\overline{[f]} \bar{m} l_{123} l_{4} L}\left(\omega_{\mathcal{\varkappa}}\right) \chi_{S T}^{[\tilde{f}] \widetilde{v} \tilde{m}}(\boldsymbol{\sigma}, \boldsymbol{\tau}) \\
\Psi_{K_{5} K_{4}}^{[\bar{f}] \bar{m} \bar{v} l_{123} l_{4} L}(\omega)=\sum_{l_{1} l_{2} l_{12} l_{3} K_{3}} C_{K_{4} l_{123}}^{[\bar{f}] \bar{v} \bar{v}}\left(l_{1} l_{2} l_{12} l_{3} K_{3}\right) \Psi_{K_{3} K_{4} K_{5}}^{l_{1} l_{2} l_{12} l_{3} l_{123} l_{4} L}(\omega)
\end{array}
$$

Five-body hyperspherical functions $\Psi_{K_{5} K_{4}}^{[\bar{f}]} \bar{v} \bar{v} l_{123} l_{4} L(\omega)$, symmetrized with respect to four identical particles, are constructed in (4+1) configuration by the use of the Parentage Scheme of Symmetrization [1]. In formula (7) $[\hat{f}]$ represents a Yong scheme of the irreducible representation of the group $S_{4}$ and is obtained from the Young scheme corresponding to the five-body function $\Psi_{K_{5} L}^{[f](m) v}$ by removing the square corresponding to the fifth particle, $\bar{m}$ denotes the rows of the $[\bar{f}]$ representation, $\bar{\nu}$ is the number of $[\bar{f}]$ with given $\mathrm{K}$ and $\mathrm{L}, C_{K_{4 l_{123}}}^{[\overline{\mathrm{m}} \bar{\nu}}$ are four particle symmetrization coefficients obtained in [1].

The binding energy of $H e_{\lambda}^{5}$ is calculated by considering the following quantum numbers in (6):

$$
K_{4}=l_{123}=l_{4}=L=0 ; \quad K_{5}=0,2
$$

When $K_{4}=0$, function (6) is symmetric with respect to nucleon permutations and can be written as: 


$$
\Psi(\mathbf{p}, \mathbf{q}, \mathbf{r}, \mathbf{s}\{\boldsymbol{\sigma}, \boldsymbol{\tau}\})=\varphi_{00}^{[4]}(\varkappa) \Gamma_{00}^{[4]}\left(\omega_{\varkappa}, \boldsymbol{\sigma}, \boldsymbol{\tau}\right)+\varphi_{20}^{[4]}(\varkappa) \Gamma_{20}^{[4]}\left(\omega_{\varkappa}, \boldsymbol{\sigma}, \boldsymbol{\tau}\right)
$$

Taking into consideration (9) in (4) the following system of one-dimensional integral equations is obtained:

$$
\begin{aligned}
& \varkappa^{5}\left(\varkappa^{2}+\bar{\varkappa}_{0}^{2}\right) \varphi_{0}(\varkappa)=-\frac{2 \mathrm{M}}{\hbar^{2}} \int d \varkappa^{\prime} \varkappa^{\prime 6} \varphi_{0}\left(\varkappa^{\prime}\right) \int \rho d \rho J_{5}\left(\varkappa_{\rho}\right) J_{5}\left(\varkappa^{\prime} \rho\right)\left\langle\Gamma_{00}\left(\omega_{\varkappa},\right) \boldsymbol{\sigma}, \boldsymbol{\tau}|U| \Gamma_{00}\left(\omega_{\varkappa}, \boldsymbol{\sigma}, \boldsymbol{\tau}\right)\right\rangle+ \\
& +\frac{2 \mathrm{M}}{\hbar^{2}} \int d \mathcal{\varkappa}^{\prime} \mathcal{\varkappa}^{\prime 6} \varphi_{2}\left(\mathcal{\varkappa}^{\prime}\right) \int \rho d \rho J_{5}\left(\varkappa_{\rho}\right) J_{7}\left(\mathcal{\varkappa}^{\prime} \rho\right)\left\langle\Gamma_{00}\left(\omega_{\varkappa}, \boldsymbol{\sigma}, \boldsymbol{\tau}\right)|U| \Gamma_{20}\left(\omega_{\varkappa}, \boldsymbol{\sigma}, \boldsymbol{\tau}\right)\right\rangle ; \\
& \mathcal{\varkappa}^{5}\left(\mathcal{\varkappa}^{2}+\overline{\mathcal{H}}_{0}^{2}\right) \varphi_{2}(\varkappa)=-\frac{2 \mathrm{M}}{\hbar^{2}} \int d \mathcal{\varkappa}^{\prime} \mathcal{\varkappa}^{\prime 6} \varphi_{2}\left(\mathcal{\varkappa}^{\prime}\right) \int \rho d \rho J_{7}\left(\mathcal{\varkappa}_{\rho}\right) J_{7}\left(\mathcal{\varkappa}^{\prime} \rho\right)\left\langle\Gamma_{20}\left(\omega_{\varkappa}, \boldsymbol{\sigma}, \boldsymbol{\tau}\right)|U| \Gamma_{20}\left(\omega_{\varkappa}, \boldsymbol{\sigma}, \boldsymbol{\tau}\right)\right\rangle+ \\
& +\frac{2 \mathrm{M}}{\hbar^{2}} \int d \varkappa^{\prime} \varkappa^{\prime 6} \varphi_{0}\left(\varkappa^{\prime}\right) \int \rho d \rho J_{7}\left(\varkappa_{\rho}\right) J_{5}\left(\varkappa^{\prime} \rho\right)\left\langle\Gamma_{20}\left(\omega_{\varkappa}, \boldsymbol{\sigma}, \boldsymbol{\tau}\right)|U| \Gamma_{00}\left(\omega_{\varkappa}, \boldsymbol{\sigma}, \boldsymbol{\tau}\right)\right\rangle ;
\end{aligned}
$$

For the central, spin-isospin independent nucleon-nucleon (NN) and hyperon-nucleon $(\lambda N)$ potentials matrix elements in system (10) can be written as:

$$
\begin{aligned}
& \left\langle\Gamma_{\mathrm{K}_{5} \mathrm{~L}}\left(\omega_{\mathcal{\varkappa}}, \boldsymbol{\sigma}, \boldsymbol{\tau}\right)|U| \Gamma_{\mathrm{K}_{5}{ }^{\prime} \mathrm{L}^{\prime}}\left(\omega_{\varkappa}, \boldsymbol{\sigma}, \boldsymbol{\tau}\right)\right\rangle= \\
& \left.=\left\langle\Psi_{K_{5} L}^{[4]}\left(\omega_{\varkappa}\right)\right| 6 U_{N N}\left(\left|\boldsymbol{r}_{i}-\boldsymbol{r}_{j}\right|\right)+4 U_{\lambda N}\left(\left|\boldsymbol{r}_{\boldsymbol{l}}-\boldsymbol{r}_{\boldsymbol{n}}\right|\right)\right)\left|\Psi_{K_{5}{ }^{\prime} L^{\prime}}^{[4]}\left(\omega_{\varkappa}{ }^{\prime}\right)\right\rangle
\end{aligned}
$$

Multiple integration with the hyper-angles in $\lambda N$ matrix element is avoided by transitioning from $(4+1)$ to $(3+2)$ configuration (transformation P). Corresponding Kinematic Rotation Coefficients (KRC) were introduced in [1]. In (3+2) configuration $\left|\boldsymbol{r}_{\boldsymbol{l}}-\boldsymbol{r}_{\boldsymbol{n}}\right|$ depends only on one hyper-angle, namely:

$$
\left|\boldsymbol{r}_{\boldsymbol{l}}-\boldsymbol{r}_{\boldsymbol{n}}\right|=\sqrt{\frac{M+M_{n}}{M M_{n}}} \rho \cos \gamma(12) .
$$

To calculate $N N$ matrix element, first transition from $(4+1)$ to $(3+1)$ configuration and then rotation $12 \leftrightarrow 45$ (transformation $P^{\prime}$ ) [1] should be performed. Consequently, the matrix element (11) can be written as:

$$
\begin{aligned}
& \left\langle\Gamma_{\mathrm{K}_{5} \mathrm{~L}}\left(\omega_{\varkappa}, \boldsymbol{\sigma}, \boldsymbol{\tau}\right)|U| \Gamma_{\mathrm{K}_{5}{ }^{\prime} \mathrm{L}^{\prime}}\left(\omega_{\varkappa}, \boldsymbol{\sigma}, \boldsymbol{\tau}\right)\right\rangle=\sum_{\bar{\alpha}}\left\langle\Psi_{K_{5} L}^{\bar{\alpha}}\left(\omega_{\varkappa}\right)|U| \Psi_{K_{5} L^{\prime}}^{\bar{\alpha}}\left(\omega_{\varkappa}{ }^{\prime}\right)\right\rangle \\
& U=6\langle\bar{\alpha} \mid \alpha\rangle_{K_{5 L}}^{P^{\prime}}\langle\bar{\alpha} \mid \alpha\rangle_{K_{5} L}^{P^{\prime}} U_{N N}(\sqrt{2} \rho \cos \gamma)+4\langle\bar{\alpha} \mid \alpha\rangle_{K_{5 L}}^{P}\langle\bar{\alpha} \mid \alpha\rangle_{K_{5} L}^{P} U_{\Lambda \mathrm{N}}\left(\sqrt{\frac{M+M_{\Lambda}}{M M_{\Lambda}}} \rho \cos \gamma\right),
\end{aligned}
$$

where $\alpha \equiv\left([\bar{f}] \bar{m} \bar{v} l_{123}, l_{4,} K_{3}, K_{4}\right),\langle\bar{\alpha} \mid \alpha\rangle_{K_{5 L}}^{P^{\prime}}$ and $\langle\bar{\alpha} \mid \alpha\rangle_{K_{5 L}}^{P}$ are KRC obtained in [1].

\section{Finding binding energies and mean square distances}

Wave functions $\varphi_{0}(\varkappa), \varphi_{2}(\varkappa)$ and the binding energies of $H e_{\lambda}^{5}$ are obtained by implementing equations (13) and (14) in (10) and solving it for different potentials. Nuclear interactions are described by a) Volkov [7] and b) Baker [8] potentials.
a) $\quad V_{N N}^{\text {Volkov }}(r)=144.86 e^{-\left(\frac{r}{0.82}\right)^{2}}-83.34 e^{-\left(\frac{r^{2}}{1.6}\right)}(15)$
b) $\quad V_{N N}^{B a k e r}(r)=-51.5 e^{-\left(\frac{r}{1.6}\right)^{2}}$

Both potentials give good convergence for the binding energy of $\mathrm{He}^{4}$ and the mean square distance between $\lambda$ particle and the center of mass of $H^{3}: \varepsilon_{K_{4}=0}^{\text {Volkov }}=-27.92 \mathrm{MeV} ; \varepsilon_{K_{4=0,4}}^{\text {Volkov }}=-28.23 \mathrm{MeV}$; 
$\varepsilon_{K_{4=0}}^{\text {Baker }}=-29.65 \mathrm{MeV} ; \varepsilon_{K_{4=0,4}}^{\text {Baker }}=-29.96 \mathrm{MeV} ; R_{\lambda-H^{3}, K_{4}=0}^{\text {Volkov }}=1.54 \mathrm{fm} ; R_{\lambda-H^{3} K_{4=0,4}}^{\text {Volkov }}=1.47 \mathrm{fm}$ $R_{\lambda-H^{3}, K_{4}=0}^{\text {Baker }}=1.39 \mathrm{fm} ; R_{\lambda-H^{3}}^{\text {Baker }}=1.32 \mathrm{fm}$. The obtained results demonstrate that the expansion (9) and taking into account only $K_{4=0}$ for $H e_{\lambda}^{5}$ is acceptable. Hyperon- nucleon interactions are described by attractive potentials of a) Kolesnikov-Chernov [9] and the potential with a soft repulsion b) Ogawa [10] which describes the phases of $\Lambda \mathrm{N}$ scattering and binding energy of hypertritium very well [11].

$$
\begin{aligned}
& \text { a) } V_{\Lambda \mathrm{N}}(r)=-235(\gamma / \pi)^{2} e^{-\gamma r^{2}} \gamma=2.46 \quad \text { (17) } \\
& \text { b) } V_{\Lambda \mathrm{N}}(r)=-V_{2 \pi}\left(\frac{0.715}{r}\right) e^{\left(-\frac{r}{0.715}\right)}-V_{k}\left(\frac{0.339}{r}\right) e^{\left(-\frac{r}{0.339}\right)}+V_{\omega}\left(\frac{0.252}{r}\right) e^{\left(-\frac{r}{0.252}\right)}
\end{aligned}
$$

Solving the system (10) with attractive $(\lambda N)$ potential (17) and taking into account the first harmonic $K_{5}=0$ gives $B_{\lambda}=4.9 \mathrm{MeV}$ for the binding energy of $\lambda$ particle in $\mathrm{He}_{\lambda}^{5}$ which is larger than experimental result $B_{\lambda}^{\text {exp }}=3.12 \mathrm{MeV}$ [11]. However, the obtained result agrees with the calculations based on two-particle $\lambda-\alpha$ model of $H e_{\lambda}^{5}$. Solving the system (10) by the use of the potential (18) and taking into account first two harmonics $K_{5}=0$ and $K_{5}=2$ gives the following results for the $H e_{\lambda}^{5}$ :

$\varepsilon_{K_{5}=0}^{\text {Volkov }}=-29.87 \mathrm{MeV} ; \varepsilon_{K_{5}=0,4}^{\text {Volkov }}=-31.59 \mathrm{MeV} ; R_{\lambda-\alpha, K_{5=0}}^{\text {Volkov }}=4.21 \mathrm{fm} ; R_{\lambda-\alpha, K_{5=0,4}}^{\text {Volkov }}=3.47 \mathrm{fm}$ $\varepsilon_{K_{5=0}}^{\text {Baker }}=-31.75 \mathrm{MeV} ; \varepsilon_{K_{5=0,4}}^{\text {Baker }}=-33.44 \mathrm{MeV} ; R_{\lambda-\alpha, K_{5=0}}^{\text {Baker }}=3.99 \mathrm{fm} ; R_{\lambda-\alpha, K_{5=0,4}}^{\text {Baker }}=3.21 \mathrm{fm}$

The obtained results demonstrate good convergence for the binding energies and the mean square distances. However, calculated binding energies of $\lambda$ particle in $\mathrm{He}_{\lambda}^{5} \quad B_{\lambda}^{\text {Volkov }}=3.67 \mathrm{MeV}$ and $B_{\lambda}^{\text {Baker }}=3.79 \mathrm{MeV}$ are larger than experimental results [11].

\section{Conclusions}

The five-particle hypernucleus $H e_{\lambda}^{5}$ as a system of four nucleons and one hyperon is investigated by the use of the Hyperspherical Function Method in momentum representation. The binding energies of $H e^{4}$ and $H e_{\lambda}^{5}$ and the mean square $\lambda-H^{3}$ and $\lambda-\alpha$ distances for $H e^{4}$ and $H e_{\lambda}^{5}$ are calculated by the use of the different nucleon-nucleon and hyperon-nucleon potentials. The obtained results demonstrate good convergence taking into account first two harmonics.

The comparison of the results obtained by the use of different potentials demonstrates that increasing repulsion in both $(N N)$-and $(\lambda N)$ - potentials, along with taking into account additional harmonics, may lead to decrease in the calculated values of the binding energies and provide satisfactory agreement with existing experimental data.

\section{References}

1. L.L. Margolin, J. Phys. Conf. Ser. 343 (2012).

2. L.L.Margolin, Sh.M.Tsiklauri. e-Print ArXiv:math-phys/0710.3079, (2007)

3. R. Jibuti, N.Krupennikova, L.Sarkisyan-Margolin, Sh.M.Tsiklauri, Yad.Fiz. 44, 349 (1986)

4. N.B.Krupennikova. Yad.Fiz. 32, 940 (1990)

5. N.Krupennikova, L.Chachanidze-Margolin, Sh. Tsiklauri. Phys. of Atom. Nucl. 56, 1327 (1993)

6. Xi. Wang at al. Progr. Theor. Phys. 76, 865 (1986)

7. A.B.Volkov. J.Nucl.Phys. 74, 33 (1965)

8. G.A. Baker. Phys. Rev. 5 (1962) 1754

9. N.N. Kolesnikov. S.M. Chernov. Soviet J. Nucl. Phys. 23, 968 (1980)

10. K. Ogawa at al. Progr. Theor. Phys 63, 533 (1980)

11. M. Juric at al. Nucl Phys. B52 1 (1973)

12. N. Krupennikova. Soviet J. Nucl. Phys. 51 (1990) 62 